\title{
Relapsing Uveitis in Association with Presumed Sinus Histiocytosis
}

\author{
Mizue Nakashima ${ }^{1,2}$, Yoshie Matsui ${ }^{1}$, and Setsu Kobayashi ${ }^{3}$ \\ ${ }^{1}$ Department of Ophthalmology, Kyoto Katsura Hospital, Kyoto, Japan; ${ }^{2}$ Department of \\ Ophthalmology, Saga University Faculty of Medicine, Saga, Japan; ${ }^{3}$ Department of \\ Dermatology, Kyoto Katsura Hospital, Kyoto, Japan
}

Japanese Journal of Ophthalmology 2006;50:484-486

The affiliations should be shown as follows:

Mizue Nakashima ${ }^{1,2}$, Yoshie Matsui ${ }^{1}$, and Setsu Kobayashi ${ }^{3}$

${ }^{1}$ Department of Ophthalmology, Kyoto Katsura Hospital, Kyoto, Japan; ${ }^{2}$ Department of Ophthalmology, Saga University Faculty of Medicine, Saga, Japan; ${ }^{3}$ Department of Dermatology, Kyoto Katsura Hospital, Kyoto, Japan

Received: May 1, 2005 / Accepted: January 16, 2006

Correspondence to: Mizue Nakashima, Department of Ophthalmology, Saga University Faculty of Medicine, 5-1-1 Nabeshima, Saga 8498501, Japan 